\title{
Online Ramsey Theory for Planar Graphs
}

\author{
Šárka Petříčková \\ Department of Mathematics \\ University of West Bohemia \\ Univerzitní 8, 30614 Plzeň, Czech Republic \\ sarpet@kma.zcu.cz
}

Submitted: Dec 1, 2012; Accepted: Mar 10, 2014; Published: Mar 24, 2014

Mathematics Subject Classification: 05C15, 05D10, 91A46

\begin{abstract}
An online Ramsey game $(G, \mathcal{H})$ is a game between Builder and Painter, alternating in turns. During each turn, Builder draws an edge, and Painter colors it blue or red. Builder's goal is to force Painter to create a monochromatic copy of $G$, while Painter's goal is to prevent this. The only limitation for Builder is that after each of his moves, the resulting graph has to belong to the class of graphs $\mathcal{H}$. It was conjectured by Grytczuk, Hałuszczak, and Kierstead (2004) that if $\mathcal{H}$ is the class of planar graphs, then Builder can force a monochromatic copy of a planar graph $G$ if and only if $G$ is outerplanar. Here we show that the "only if" part does not hold while the "if" part does.
\end{abstract}

Keywords: Ramsey theory; Online Ramsey games; Planar graphs; Outerplanar graphs; Game theory; Builder and Painter

\section{Introduction}

For a fixed graph $G$ and a class of graphs $\mathcal{H}$ such that $G \in \mathcal{H}$, an online Ramsey game $(G, \mathcal{H})$, defined by Grytczuk, Hałuszczak, and Kierstead [5], is a game between Builder and Painter with the following rules. The game starts with the empty graph on infinitely many vertices. On the $i$-th turn, Builder adds a new edge to the graph created in the first $i-1$ turns so that the resulting graph belongs to $\mathcal{H}$ (we say that Builder plays on $\mathcal{H}$ ), and Painter colors this edge blue or red. Builder wins if he can always force Painter to create a monochromatic copy of $G$ (or force $G$ for short). We then say that $G$ is unavoidable on $\mathcal{H}$. A graph $G$ is unavoidable if it is unavoidable on planar graphs. On the other hand, if Painter can ensure that a monochromatic copy of $G$ is never created, then $G$ is avoidable on $\mathcal{H}$. A class of graphs $\mathcal{H}$ is self-unavoidable if every graph of $\mathcal{H}$ is unavoidable on $\mathcal{H}$.

According to Ramsey's theorem, for every $t \in \mathbb{N}$ there exists $n \in \mathbb{N}$ such that every 2-coloring of the edges of $K_{n}$ contains a monochromatic copy of $K_{t}$. Thus, without 
restricting to $\mathcal{H}$, Builder would always win the online Ramsey game by creating sufficiently large complete graph. The size Ramsey number $r(G)$ for a graph $G$ is the minimum number of edges of a graph that contains a monochromatic copy of $G$ in every 2-coloring of its edges. The online size Ramsey number $\tilde{r}(G)$ is the minimum $m$ such that Builder can force $G$ by playing on the class of graphs with at most $m$ edges. Clearly, $\tilde{r}(G) \leqslant r(G)$ (Builder wins by presenting a graph of size $r(G)$ that contains a monochromatic copy of $G$ for any 2-edge-coloring). However, Builder may be able to win using less than $r(G)$ edges since he can adapt his strategy to Painter's coloring. One can then ask whether or not $\tilde{r}(G)=o(r(G))$. The basic conjecture in the field, attributed to Rödl by Kurek and Ruciński [9], is that $\tilde{r}\left(K_{t}\right)=o\left(r\left(K_{t}\right)\right)$. In 2009, Conlon [3] showed that $\tilde{r}\left(K_{t}\right) \leqslant 1.001^{-t}\left(r\left(K_{t}\right)\right)$ for infinitely many $t$. On the other hand, if $G$ is a path or a cycle, then both $\tilde{r}(G)$ and $r(G)$ are linear in $|V(G)|$ (see [1], [6], [7]).

Butterfield et al. [2] studied Online Ramsey games played on the class $\mathcal{S}_{k}$ of graphs with maximum degree at most $k$. The authors introduce an online degree Ramsey number $\tilde{r}_{\triangle}(G)$ as the least $k$ for which $G$ is unavoidable on $\mathcal{S}_{k}$.

Online Ramsey games played on various classes of graphs were studied by Grytczuk et al. [5]. They proved that the class of $k$-colorable graphs as well as the class of forests are self-unavoidable. (It was later shown by Kierstead and Konjevod [8] that the $k$-colorable graphs are self-unavoidable even if Painter uses more colors.) Various games played on planar graphs were investigated in [5]. It was shown, for example, that every cycle, as well as the graph $K_{4}-e$, is unavoidable on planar graphs. They made the following conjecture:

Conjecture ([5]). The class of graphs unavoidable on planar graphs is exactly the class of outerplanar graphs.

Here we show that the conjecture is only partially true. In particular, it is true that the class of outerplanar graphs is a subclass of the class of graphs unavoidable on planar graphs.

Theorem 1. Every outerplanar graph is unavoidable on planar graphs.

However, we show that there exists an infinite family of planar but not outerplanar graphs which are unavoidable on planar graphs. Let $\theta_{i, j, k}$ denote the union of three internally disjoint paths of lengths $i, j, k$, respectively.

Theorem 2. The graph $\theta_{2, j, k}$ is unavoidable for even $j, k$.

The paper is organized as follows. In Section 2, we introduce notation. Section 3 gives a proof of Theorem 1, and Section 4 gives a proof of Theorem 2 .

\section{Notation}

In this section, we first mention several notions that are particularly important for the next discussion. Besides these, we follow standard graph theory terminology (see Diestel [4]). 

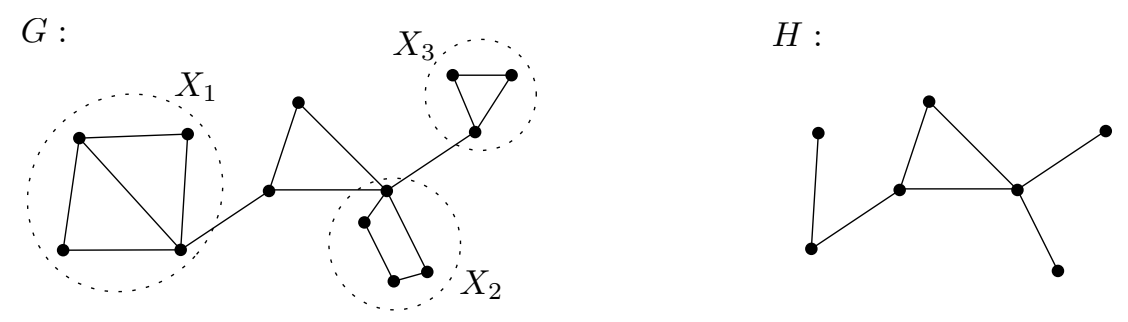

Figure 1: Graphs $G$ and $H$ such that $G \triangleright H$.

All graphs considered here are simple and undirected. For a graph $G$, the set of vertices is denoted by $V(G)$ and the set of edges by $E(G)$. The length of a path is the number of its edges. If we replace an edge $e$ of $G$ with a path of length $k+1$ (i.e. we place $k$ vertices of degree 2 on $e$ ), then we say that $e$ is subdivided $k$-times. For a fixed graph $G$, a copy $H$ of $G$ is a graph isomorphic to $G$ with $V(G) \cap V(H)=\emptyset$. For two graphs $G_{1}=\left(V_{1}, E_{1}\right)$ and $G_{2}=\left(V_{2}, E_{2}\right)$, their union is the graph $G_{1} \cup G_{2}=\left(V_{1} \cup V_{2}, E_{1} \cup E_{2}\right)$. When we say that a graph is a disjoint union of $G_{1}$ and $G_{2}$, we are automatically assuming that $V_{1} \cap V_{2}=\emptyset$. A planar graph is a graph that can be drawn in the plane without edge crossings. An outerplanar graph is a planar graph that can be embedded so that all its vertices belong to the boundary of the outer face. A red-blue graph is a graph with its edges colored red or blue. A red-blue graph will often use the same name as its underlying (uncolored) graph.

Let $G_{1}$ and $G_{2}$ be two disjoint graphs containing cliques $H_{1}, H_{2}\left(H_{i} \subseteq G_{i}\right)$ of size $k \geqslant 0$. Let the vertices of $H_{i}$ be labeled $v_{1}\left(G_{i}\right), \ldots, v_{k}\left(G_{i}\right)$. A $k$-sum $G_{1} \oplus_{k} G_{2}$ of $G_{1}$ and $G_{2}$ is a graph formed from the disjoint union of $G_{1}$ and $G_{2}$ by identifying the vertex $v_{j}\left(G_{1}\right)$ with $v_{j}\left(G_{2}\right)$ for each $j=1, \ldots, k$. To simply notation, we write $G_{1} \oplus G_{2}$ if $k \leqslant 2$. Note that $G_{1} \oplus G_{2}$ does not specify the appending cliques, and so it is not a well-defined operation. However, if $k=1$, then we can make this notation precise and specify the appending vertex $v$ by writing $G_{1} \oplus_{v} G_{2}$ (which we will do often). For $k=2$, we sometimes write $G_{1} \oplus_{e} G_{2}$, where $e\left(G_{i}\right)$ is a non-oriented edge $v_{1}\left(G_{i}\right) v_{2}\left(G_{i}\right)$ (the resulting graph is again not always unique). Also, we abbreviate $\left(\left(G_{1} \oplus G_{2}\right) \oplus \ldots\right) \oplus G_{n}$ by $G_{1} \oplus G_{2} \oplus \cdots \oplus G_{n}$.

Let $G$ be a graph, $H$ a subgraph of $G$. If there exist planar graphs $X_{1}, \ldots, X_{n}$ such that $G=H \oplus X_{1} \oplus \cdots \oplus X_{n}$, then we say that $G$ is reducible to $H$, and we write $G \triangleright H$. It is a well known fact that for $k \leqslant 2$, a $k$-sum of two planar graphs is planar, thus the following holds:

Remark 3. If $H$ is a planar graph, and a graph $G$ is reducible to $H$, then $G$ is planar.

Informally, $G$ is reducible to $H$ if $G$ can be formed from $H$ by successively "appending" planar graphs on edges/vertices. So, Remark 3 says that if the starting graph $H$ is planar, then so is $G$.

Consider an online Ramsey game on planar graph. A strategy (for Builder) $\mathbf{X}$ is a finite sequence of rules that tell Builder how to move on any given turn of the game, no matter how Painter plays. If a monochromatic copy of the target graph $G$ arises, 
the game ends and Builder wins (provided that the final red-blue graph is planar). The output graph of strategy $\mathbf{X}$ is then the final red-blue graph with a fixed monochromatic copy of $G$, called a winning copy (of $G$ by $\mathbf{X}$ ) and denoted simply by $G$ if no confusion can arise. This winning copy adopts all notation from the target graph. For example, for a target graph $G$ with vertices $u, v$ and a cycle $C$, the two corresponding vertices and the corresponding cycle are again denoted by $u, v, C$ in the chosen winning copy $G$. If Builder always wins when following strategy $\mathbf{X}$, then we say that $G$ is unavoidable by strategy $\mathbf{X}$. The set of all output graphs of a strategy $\mathbf{X}$ is denoted $\mathcal{X}$ (the calligraphic version of the name of the strategy).

\section{Outerplanar graphs}

In this section we show that every outerplanar graph is unavoidable on the class of planar graphs. The idea behind our proof is based on the inductive proof of the self-unavoidability of forests presented by Grytczuk et al. [5]. Suppose that Builder's goal is to force a forest $T$. We can assume that $T$ is a tree (since every forest is contained in some tree). Choose a leaf $u$ of $T$, let $v$ be the neighbor of $u$ in $T$, and let $T^{\prime}=T-u$. Builder forces $2|T|-1$ monochromatic copies of $T^{\prime}$ (where the corresponding final graphs are pairwise disjoint), from which at least $|T|$ are of the same color, say blue. On those copies, Builder builds a new copy of $T$ by adding edges between copies of $v$. If any one of the new added edges is blue, then that edge and a blue copy of $T^{\prime}$ appended to one of its endpoints form a blue copy of $T$. Otherwise, those edges form a red copy of $T$. We will call this strategy the tree strategy.

Since trees are planar, the tree strategy shows that forests are unavoidable (on planar graphs). Moreover, a generalized version of the tree strategy can be used for forcing a graph formed from a tree $T$ by appending a copy of an unavoidable graph $G$ to each vertex of $T$. Before presenting this strategy we need some notation.

Let $T$ be a tree on vertices $v_{1}(T), \ldots, v_{n}(T)$, and let $G$ be a graph with an arbitrary vertex labeled by $v$. The ordered triple $(T, G, v)$ denotes the graph $T \oplus_{v_{1}} G_{1} \oplus_{v_{2}} \cdots \oplus_{v_{n}} G_{n}$, where for $i=1, \ldots, n, G_{i}$ is a copy of $G$ and $v_{i}\left(G_{i}\right) \in V\left(G_{i}\right)$ is the copy of $v$. We refer to the identified vertices $v_{i}(T)=v_{i}(G)$ in $(T, G, v)$ by $v_{i}$. Next, let $\mathcal{S}$ be any set of red-blue graphs $X$ such that each has a fixed monochromatic copy $G_{X}$ of $G$. Let $A$ be a red-blue graph with a fixed monochromatic subgraph $(T, G, v)=T \oplus_{v_{1}} G_{1} \oplus_{v_{2}} \cdots \oplus_{v_{n}} G_{n}$. We say that $A$ is $(T, \mathcal{S})$-reducible if $A \triangleright T \oplus_{v_{1}} X_{1} \oplus_{v_{2}} \cdots \oplus_{v_{n}} X_{n}$, where for each $i \in[n]$ either $X_{i}$ is a monochromatic copy of $G$, or $X_{i} \in \mathcal{S}$ such that $G_{i}=G_{X_{i}}$. In our proofs we take $\mathcal{S}$ to be the set of all final graphs of some strategy. For example, the set of all output graphs $\mathcal{X}$ of a strategy $\mathbf{X}$ is a set of red-blue graphs, each with a fixed monochromatic copy of $G$, and so, for any given tree $T$, we can talk about $(T, \mathcal{X})$-reducible graphs.

Suppose that $G$ is unavoidable by strategy $\mathbf{X}$. We consider the following Builder's strategy for forcing a monochromatic copy of $(T, G, v)$. 

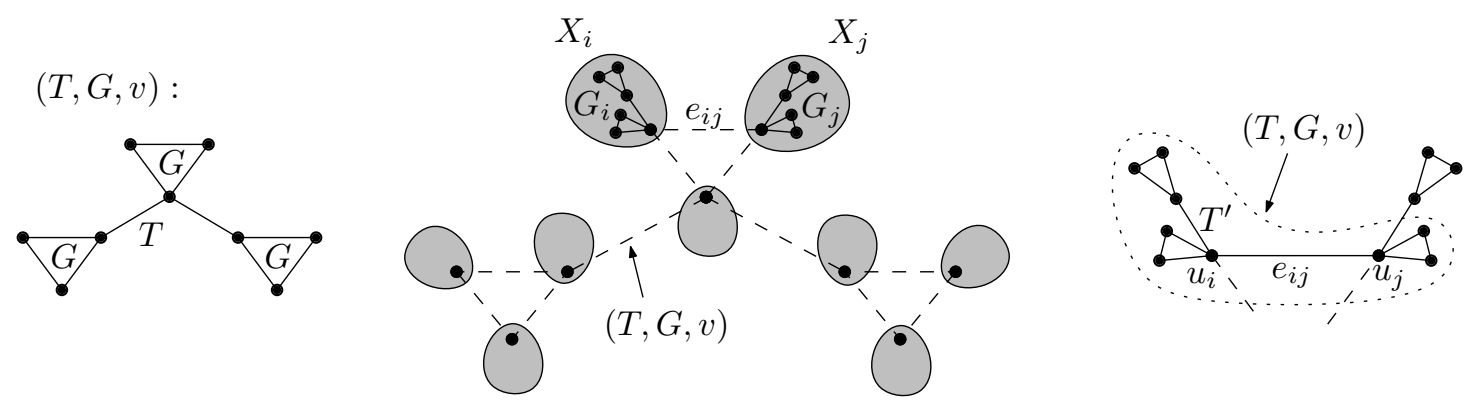

Figure 2: Forcing a monochromatic copy of $(T, G, v)$, where $T$ is a path of length $2, G$ a triangle, and $v$ is an arbitrary vertex of $V(G)$.

strategy $\mathbf{A}(T, G, v, \mathbf{X})$

Let $n=|V(T)|, k=|V((T, G, v))|$.

1. If $n=1$, call strategy $\mathbf{X}$ and stop.

2. If $n>1$, choose a leaf $u$ of $T$, set $T^{\prime}=T-u$, and call strategy $\mathbf{A}\left(T^{\prime}, G, v, \mathbf{X}\right)$ $(2 k-1)$-times. Choose $k$ copies $H_{1}, \ldots, H_{k}$ of $\left(T^{\prime}, G, v\right)$ of the same color, and in $i$ th of them label the vertex that corresponds to the neighbor of $u$ in $T$ by $u_{i}$. Add an edge $e_{i j}=u_{i} u_{j}$ if and only if $v_{i} v_{j}$ is an edge in $(T, G, v)$.

To prove that $(T, G, v)$ is unavoidable by strategy $\mathbf{A}(T, G, v, \mathbf{X})$, we have to ensure that no matter how Painter plays, a monochromatic copy of the target graph $(T, G, v)$ eventually appears, and that the final graph is planar. Both parts are shown below using induction and reduction arguments that rely on Remark 3.

Lemma 4. Let $T$ be a tree, $G$ a graph, and $v$ a vertex of $V(G)$. If $G$ is unavoidable by strategy $\mathbf{X}$, then $(T, G, v)$ is unavoidable by strategy $\mathbf{A}(T, G, v, \mathbf{X})$, and every graph $A$ of $\mathcal{A}(T, G, v, \mathbf{X})$ is $(T, \mathcal{X})$-reducible.

Proof. We use all the notation introduced in strategy $\mathbf{A}$. The proof is by induction on the number $n$ of vertices of $T$. If $n=1$, then $(T, G, v)=G$, which is unavoidable by strategy $\mathbf{X}$ by the assumption. Since $\mathcal{A}(T, G, v, \mathbf{X})=\mathcal{X}$, the graph $A$ is $(T, \mathcal{X})$-reducible. Now let $n>1$. The following two cases can arise.

Case 1: All edges $e_{i j}$ are red. These edges form a red $(T, G, v)$. Every final graph for forcing $H_{i}$ is planar by the induction hypothesis. Observe that each such graph is appended to $(T, G, v)$ by one vertex only. Thus, $A$ is reducible directly to $(T, G, v)$, and hence is $(T, \mathcal{X})$-reducible, which proves the planarity as well as the second part of the claim. See Figure 2.

Case 2: Some edge $e_{i j}$ is blue. The graph $H_{i}$, the edge $e_{i j}$, and one copy of $G$ contained in $H_{j}$ form a blue $(T, G, v)$. The graph $A$ is planar by previous discussion, so the first part of the claim is complete. Let $A_{i}, A_{j} \in \mathcal{A}\left(T^{\prime}, G, v, \mathbf{X}\right)$ be subgraphs of $A$ that were used for forcing $H_{i}$ and $H_{j}$, respectively. By the induction hypothesis, $A_{i}$ is $\left(T^{\prime}, \mathcal{X}\right)$-reducible. 

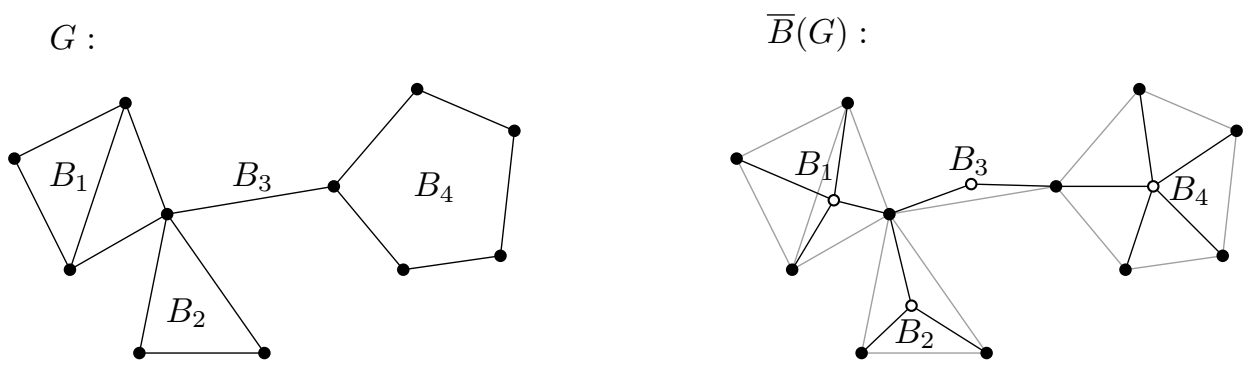

Figure 3: A graph $G$ and its complete block graph $\bar{B}(G)$.

Similarly $A_{j}$ is $\left(T^{\prime}, \mathcal{X}\right)$-reducible, and therefore is $\left(u^{\prime}, \mathcal{X}\right)$-reducible. Since the rest of $A$ is reducible to $e_{i j}$, and $e_{i j}$ shares with each of $H_{i}$ and $H_{j}$ only one vertex (the vertex $u_{i}$ and $u_{j}$, respectively), we get the second part of the claim.

A block is a maximal 2-connected subgraph. For a graph $G$ with a vertex set $V=$ $\left\{v_{1}, \ldots, v_{k}\right\}$ and blocks $B_{1}, \ldots, B_{l}$, the complete block graph $\bar{B}(G)$ is a graph on $V \cup$ $\left\{B_{1}, \ldots, B_{l}\right\}$ formed by the edges $v_{i} B_{j}$ with $v_{i} \in V\left(B_{j}\right)$ (see Figure 3). Notice that $\bar{B}(G)$ can be obtained from the block graph $B(G)$ of $G$ by adding edges with one endpoint of degree 1 , and thus, $\bar{B}(G)$ is a tree for every connected graph $G$.

Remark 5. The union of an outerplanar graph $G$ and its complete block graph $\bar{B}(G)$ is planar.

Let $H$ be an outerplanar graph. The weak dual $H^{*}$ of $H$ is the graph obtained from the plane dual of $H$ by removing the vertex that corresponds to the outer face of $H$. It is easy to see that $H^{*}$ is a forest, which is a tree whenever $H$ is 2-connected. If there exists a vertex $r \in V\left(H^{*}\right)$ such that $H^{*}$ rooted in $r$ (denoted by $H^{*}(r)$ ) is a full binary tree, then we call $H$ a full outerplanar graph. The height $h(H)$ of a full outerplanar graph $H$ is the number of levels in its full binary tree $H^{*}(r)$. The edge of a full outerplanar graph $H$ incident to the face that corresponds to $r$, as well as to the the outer face, is the central edge $e_{H}$ of $H$ (see Figure 4, left). For the sake of convenience, a graph that consists of a single edge is also considered to be full outerplanar. Its height is then defined to be 0 and its central edge is the only edge of the graph.

Lemma 6. For every outerplanar graph $G$ there exists a full outerplanar graph $H$ such that $G \subseteq H$.

Proof. Let $G_{T}$ be an almost triangulation of $G$, i.e. an outerplanar graph formed by triangulating the inner faces of $G$. The maximum degree of $G_{T}^{*}$ is at most 3 , and there exists a vertex $r \in V\left(G_{T}^{*}\right)$ of degree 1 or 2 . Let $H^{*}(r)$ be a full binary tree of height $h\left(G_{T}^{*}(r)\right)$ containing $G_{T}^{*}$. The graph $H$ is then the desired full outerplanar graph.

Recall that for a tree $T$ with $n$ vertices and $m$ edges, a graph $G$, and a vertex $v \in V(G)$, we have $(T, G, v)=T \oplus_{v_{1}} G_{1} \oplus_{v_{2}} \cdots \oplus_{v_{n}} G_{n}$. Let $H$ be a full outerplanar graph, and 
$H$ :

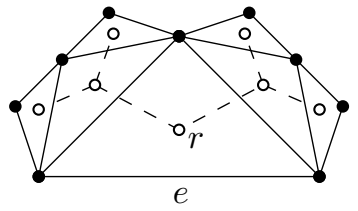

$(T, G, v, H):$

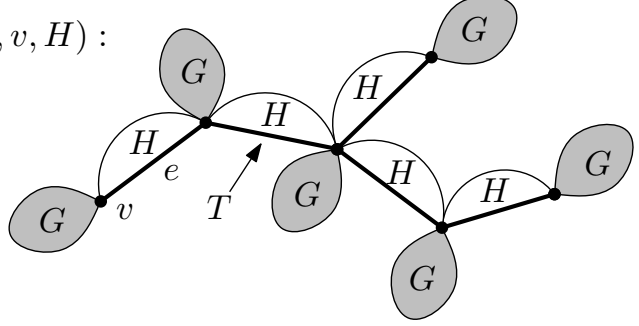

Figure 4: A full outerplanar graph $H$ with its full binary tree $H^{*}(r)$ of height 3 (left). The structure of $(T, G, v, h)$ (right).

for $i=1, \ldots, m$, let $H_{i}$ be a copy of $H$ with the central edge $e_{i}\left(H_{i}\right)$. Then we define $(T, G, v, H)$ as a graph $T \oplus_{v_{1}} G_{1} \oplus_{v_{2}} \cdots \oplus_{v_{n}} G_{n} \oplus_{e_{1}} H_{1} \oplus_{e_{2}} \cdots \oplus_{e_{m}} H_{m}$. So, $(T, G, v, H)$ is simply the graph that arises from $(T, G, v)$ if we "glue" a copy of $H$ by its central edge to every edge of $T$ (cf. Figure 4, right).

We now present a strategy $\mathbf{B}$ for forcing a monochromatic copy of $(T, G, v, H)$, assuming that $G$ is unavoidable by a strategy $\mathbf{X}$.

strategy $\mathbf{B}(T, G, v, H, \mathbf{X})$

Let $t=|V(T)|$ and $h=h(H)$.

1. If $t=1$, call strategy $\mathbf{X}$ and stop.

2. If $h=0$, call strategy $\mathbf{A}(T, G, v, \mathbf{X})$ and stop.

3. Choose a leaf $u$ of $T$ and call its neighbor $u^{\prime}$. Call strategy $\mathbf{B}\left(T^{\prime}, G^{\prime}, v^{\prime}, H^{\prime}, \mathbf{X}^{\prime}\right)$, where

- $T^{\prime}=\bar{B}(T, G, v, H)$,

- $G^{\prime}=(T-u, G, v, H)$,

- $v^{\prime}$ is the vertex of $T-u \subseteq G^{\prime}$ that corresponds to $u^{\prime}$,

- $H^{\prime}$ is the full outerplanar graph of height $h-1$,

- $\mathbf{X}^{\prime}=\mathbf{B}(T-u, G, v, H, \mathbf{X})$.

Let $\left\{u_{1}, \ldots, u_{k}\right\}$ be the vertex set of $(T, G, v, H)$, and thus also a subset of a vertex set of $\bar{B}(T, G, v, H)=T^{\prime}$. Adopt this notation to the subgraph $T^{\prime}$ of the winning copy $\left(T^{\prime}, G^{\prime}, v^{\prime}, H^{\prime}\right)$ found by strategy $\mathbf{B}\left(T^{\prime}, G^{\prime}, v^{\prime}, H^{\prime}, \mathbf{X}^{\prime}\right)$. Add an edge $e_{i j}=u_{i} u_{j}$ in $\left(T^{\prime}, G^{\prime}, v^{\prime}, H^{\prime}\right)$ if and only if $u_{i} u_{j}$ is an edge in $(T, G, v, H)$.

Let $\mathcal{S}$ be a set of red-blue graphs such that each $X \in \mathcal{S}$ contains a fixed monochromatic graph $G$. Then we set $\overline{\mathcal{S}}=\mathcal{S} \cup\{G \cup \bar{B}(G)\}$, where $G$ is the fixed monochromatic graph.

Theorem 1. Every outerplanar graph is unavoidable (on planar graphs). 

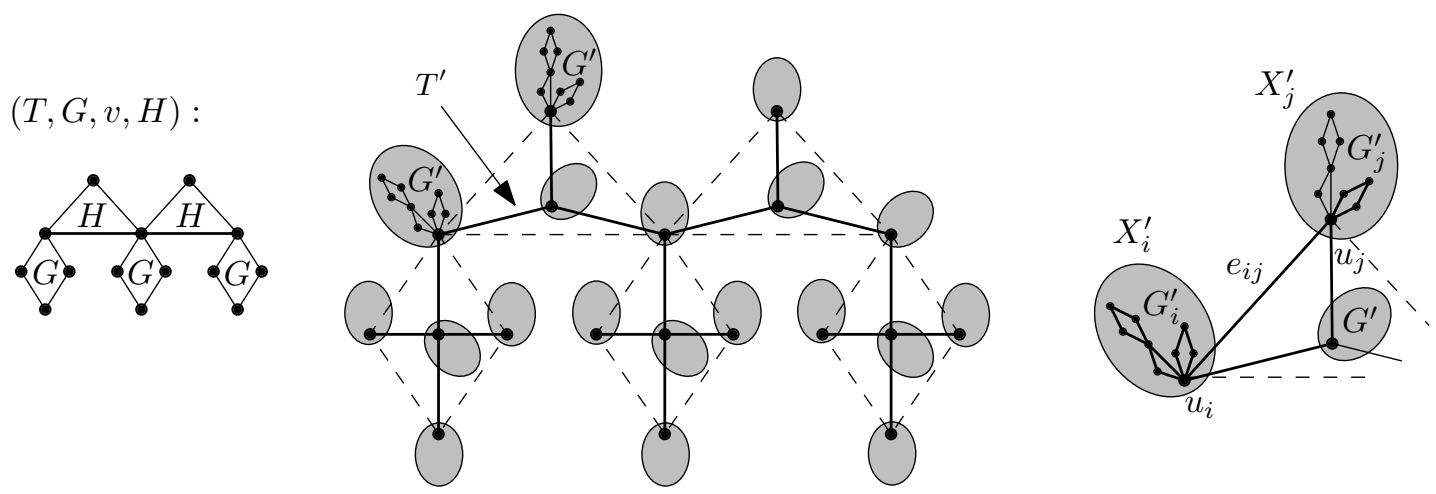

Figure 5: Forcing a monochromatic copy of $(T, G, v, H)$, where $T$ is a path of length 2, $G$ is a cycle of length $4, v$ is any vertex of $V(G)$, and $H$ is the full outerplanar graph of height 1.

Proof. We prove a stronger claim instead.

Claim. Let $T$ be a tree, $G$ an outerplanar graph, and $v \in V(G)$. If $G$ is unavoidable by strategy $\mathbf{X}$, then $(T, G, v, H)$ is unavoidable by strategy $\mathbf{B}(T, G, v, H, \mathbf{X})$, and every graph $B \in \mathcal{B}(T, G, v, H, \mathbf{X})$ is $(T, \overline{\mathcal{X}})$-reducible.

This statement implies Theorem 1 since every outerplanar graph $G$ is contained in some full outerplanar graph $H$ by Lemma 6 , which can be written as $\left(e_{H},(\{v\}, \emptyset), v, H\right)$, and is therefore unavoidable by the above claim.

We adopt all the notation used in strategy B. Let $S$ be the set of all 2-tuples $(h, t) \in$ $(\mathbb{N} \cup\{0\}) \times \mathbb{N}$. On $S$, we define the lexicographic order $\unlhd$, i.e. $\left(h_{1}, t_{1}\right) \unlhd\left(h_{2}, t_{2}\right)$ exactly when $h_{1}<h_{2}$, or $h_{1}=h_{2}$ and $t_{1} \leqslant t_{2}$ for all $h_{1}, h_{2} \in \mathbb{N} \cup\{0\}$ and $t_{1}, t_{2} \in \mathbb{N}$. The set $S$ together with the relation $\unlhd$ is linear, and we can apply induction.

We start with the basis. Suppose first that $h \geqslant 0$, and $t=1$. Then $(T, G, v, H)=G$ and the claim is trivially satisfied. Let now $h=0$, and $t \geqslant 1$. In this case we have $(T, G, v, H)=(T, G, v)$. By Lemma $4,(T, G, v)$ is unavoidable by $\mathbf{A}(T, G, v, \mathbf{X})$. So, every graph of $\mathcal{A}(T, G, v, \mathbf{X})$ is $(T, \mathcal{X})$-reducible, and thus $(T, \overline{\mathcal{X}})$-reducible since $\mathcal{X} \subseteq \overline{\mathcal{X}}$.

Suppose now that $h \geqslant 1, t \geqslant 2$. By the induction hypothesis $((h, t-1) \unlhd(h, t))$, $G^{\prime}=(T-u, G, v, H)$ is unavoidable by strategy $\mathbf{X}^{\prime}=\mathbf{B}(T-u, G, v, H, \mathbf{X})$, and every graph of $\mathcal{X}^{\prime}$ is $(T-u, \overline{\mathcal{X}})$-reducible. Since $G^{\prime}$ is unavoidable by strategy $\mathbf{X}^{\prime}$, it holds by the induction hypothesis $((h-1, t) \unlhd(h, t))$ that $\left(T^{\prime}, G^{\prime}, v^{\prime}, H^{\prime}\right)$ is unavoidable by strategy $\mathbf{B}^{\prime}=\mathbf{B}\left(T^{\prime}, G^{\prime}, v^{\prime}, H^{\prime}, \mathbf{X}^{\prime}\right)$, and every graph $B^{\prime}$ of $\mathcal{B}^{\prime}$ is $\left(T^{\prime}, \overline{\mathcal{X}^{\prime}}\right)$-reducible. Say that the winning copy $\left(T^{\prime}, G^{\prime}, v^{\prime}, H^{\prime}\right)$ in $B^{\prime}$ is blue. We distinguish the following two cases:

Case 1: All edges $e_{i j}$ are red. These edges form a red copy of $(T, G, v, H)$. The graph $B^{\prime}$ is $\left(T^{\prime}, \overline{\mathcal{X}^{\prime}}\right)$-reducible, and thus reducible to $T^{\prime}=\bar{B}(T, G, v, H)$. Since $B$ arose from $B^{\prime}$ by adding the edges forming $(T, G, v, H), B$ is reducible to $(T, G \cup \bar{B}(G), v)$, and thus $(T, \bar{X})$-reducible. 
Case 2: At least one edge $e_{i j}=u_{i} u_{j}$ is blue. The endpoints of $e_{i j}$ are connected by a path $P$ in $T^{\prime}$ of length 2 . There is a copy of $H^{\prime}$ appended along each of the edges of $P$. Those two copies of $H^{\prime}$ together with $e_{i j}$ form a full outerplanar graph $H$ of height $h$ with central edge $e_{i j}$ (see Figure 5). Let $G_{i}^{\prime}$ and $G_{j}^{\prime}$ be the blue copies of $G^{\prime}=(T-u, G, v, H)$ appended to $u_{i}$ and $u_{j}$, respectively. Then $H, G_{i}^{\prime}$, and the copy of $G$ in $G_{j}^{\prime}$ that is appended to $u_{j}$ form a blue copy of $(T, G, v, H)$. We can assume that Builder chooses this copy as the winning copy. We now prove the second part of the claim. Recall that $B^{\prime}$ is $\left(T^{\prime}, \overline{\mathcal{X}^{\prime}}\right)$-reducible. Let $X_{i}^{\prime}, X_{j}^{\prime}$ be the graphs of $\overline{\mathcal{X}^{\prime}}$ appended to $u_{i}, u_{j}$, respectively. So, $X_{i}^{\prime}$ is $(T-u, \overline{\mathcal{X}})$-reducible, and $X_{j}$ is $\left(\left\{u_{j}\right\}, \overline{\mathcal{X}}\right)$-reducible. Since the rest of the graph $B$ is reducible to $e_{i j}$, we find that $B$ is $(T, \overline{\mathcal{X}})$-reducible. See the diagram on the right in Figure 5 .

\section{Non-outerplanar graphs}

We now show that an infinite subclass of theta-graphs is unavoidable on planar graphs. Recall that a theta-graph ( $\theta$-graph) is the union of three internally disjoint paths that have the same two end vertices. We write $\theta_{i, j, k}$ for the theta-graph with paths of length $i, j, k$. For example, $K_{2,3}$ is the graph $\theta_{2,2,2}$.

Before stating the main theorem, we introduce a strategy for forcing even cycles. The unavoidability of cycles was proven in [5], but here we need the final graph to have a special type of plane embedding that we utilize in the proof of the main theorem.

Let $C$ be a cycle of even length $n$ that is unavoidable by strategy $\mathbf{X}$. If for every graph $X$ of $\mathcal{X}$ there is a plane embedding of $X$ such that

(G1) all vertices of $V(C)$ belong to the boundary of one common face, and

(G2) there exists a path $P \subset C$ of length $\frac{n}{2}$ such that all vertices of $V(P)$ lie the boundary of another face,

then we say that strategy $\mathbf{X}$ is a good strategy. The path $P$ is then called a good path in $C$.

strategy $\mathbf{C}(C)$

Let $n=|V(C)|, a=(n-1)$, and $b=\frac{n}{2}-1$.

1. Force a monochromatic path $P=v_{0} \ldots v_{a^{2}+b}$ by the tree strategy.

2. In $P$, Connect the vertices $v_{0}$ and $v_{a^{2}}$ by an edge $e$.

3. If $e$ has the other color than $P$, add the path $P^{\prime}=v_{0} v_{a} v_{2 a} \ldots v_{a^{2}}$. Otherwise add the cycle $C^{\prime}=v_{b} v_{(b+a)} v_{(b+2 a)} \ldots v_{\left(b+a^{2}\right)} v_{b}$.

Lemma 7. Let $C$ be an even cycle. Then strategy $\mathbf{C}(C)$ is a good strategy. 

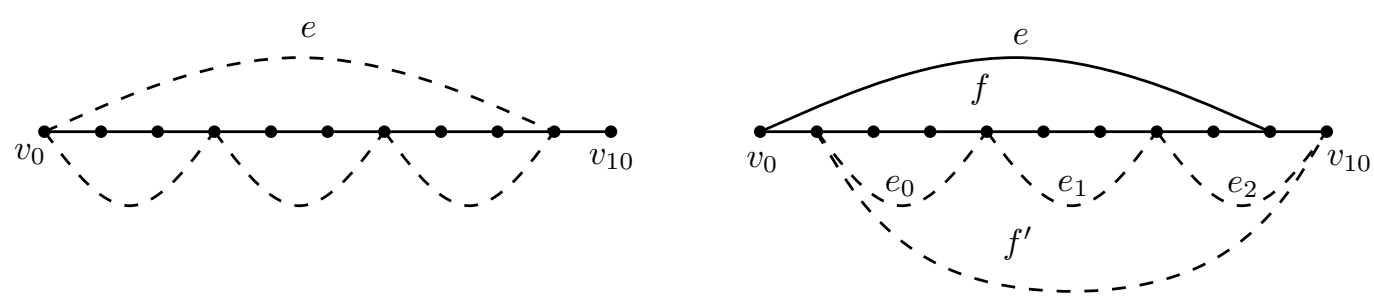

Figure 6: Forcing cycle of length 4 by strategy $C$.

Proof. We will follow the notation introduced in strategy C. We fix a planar embedding of an output graph of strategy $\mathbf{C}(C)$ as shown in Figure 6. By [5], every final graph of the tree strategy for forcing $P$ is a forest, which is reducible to (the chosen monochromatic) $P$. Assume that $P$ is blue. The following two cases can arise.

Case 1: The edge $e$ is red. If Painter colors some edge of $P^{\prime}$ blue, a blue copy of $C$ arises since there is a blue path of length $n-1$ between such two vertices. Otherwise, $P^{\prime} \cup e$ is a red cycle $C$ of length $n$. In both cases, all vertices of the monochromatic copy of $C$ belong to two common faces. See Figure 6, left.

Case 2: The edge $e$ is blue. Suppose that Painter colors some edge $e^{\prime}$ of $C^{\prime}-v_{b} v_{\left(a^{2}+b\right)}$ blue. Since each such pair is connected by a blue path of length $a=n-1$, a blue cycle of length $n$ arises. Condition (G1) is then satisfied by the face bounded by this cycle, and (G2) is satisfied by the face $f$ bounded by the cycle $v_{0} v_{a} v_{2 a} \ldots v_{a^{2}} v_{0}$, which contains a good path on $n / 2+1$ vertices if $e^{\prime}=v_{a(a-1)} v_{a^{2}}$ and on all $n$ vertices in all the other cases. Suppose now that Painter colors the edge $v_{b} v_{\left(a^{2}+b\right)}$ blue. Then the blue copy of $C$ is formed by this edge and the blue path starting at $v_{b}$, going through $e$, and ending at $v_{\left(a^{2}+b\right)}$. All of the vertices of the blue copy of $C$ belong to the outer face, and there is a good path $v_{b} v_{b-1} \ldots v_{0} v_{a^{2}}$ of length $b+1=\frac{n}{2}$ that belongs to $f$. Consider the last possibility when $C^{\prime}$ is red. Now, all of the vertices of $V(C)$ belong to the boundary of $f^{\prime}$, and all but the vertex $v_{\left(a^{2}+b\right)}$ of $V(C)$ belong to the boundary of $f$. See Figure 6 , right.

Theorem 2. The graph $\theta_{2, j, k}$ is unavoidable for even $j, k$.

Proof. For fixed $j$ and $k$, let $j^{\prime}=\frac{j}{2}, k^{\prime}=\frac{k}{2}$. We consider disjoint cycles $C_{1}, \ldots, C_{j^{\prime}+k^{\prime}+1}$ of length $k+2$. In $i$ th of them, we label an arbitrary vertex by $c_{i}$ and one of the two vertices in distance 2 from $c_{i}$ by $v_{0}\left(C_{i}\right)$ if $i \leqslant j^{\prime}+1$, and by $v_{1}\left(C_{i}\right)$ otherwise. Let $P_{1}, \ldots, P_{j^{\prime}+k^{\prime}+2}$ be paths of length $j-1$, where in each $P_{i}$, one end is labeled by $p_{i}$, and another one by $v_{0}\left(P_{i}\right)$ if $i \leqslant j^{\prime}+1$, and by $v_{1}\left(P_{i}\right)$ otherwise. Let

$$
L:=C_{1} \oplus_{v_{0}} \cdots \oplus_{v_{0}} C_{j^{\prime}+1} \oplus_{v_{0}} P_{1} \oplus_{v_{0}} \cdots \oplus_{v_{0}} P_{j^{\prime}+1}
$$

and

$$
R:=C_{j^{\prime}+2} \oplus_{v_{1}} \cdots \oplus_{v_{1}} C_{j^{\prime}+k^{\prime}+1} \oplus_{v_{1}} P_{j^{\prime}+2} \oplus_{v_{1}} \cdots \oplus_{v_{1}} P_{j^{\prime}+k^{\prime}+2} .
$$

Then we write $H$ for a graph that is formed from the disjoint union of $L$ and $R$ by identifying $p_{1}$ with $p_{j^{\prime}+k^{\prime}+2}$, and $p_{j^{\prime}+1}$ with $p_{j^{\prime}+2}$ (see Figure 7 , left). The cycle consisting of the paths $P_{1}, P_{j^{\prime}+1}, P_{j^{\prime}+2}$, and $P_{j^{\prime}+k^{\prime}+2}$ is denoted $C_{0}$. 

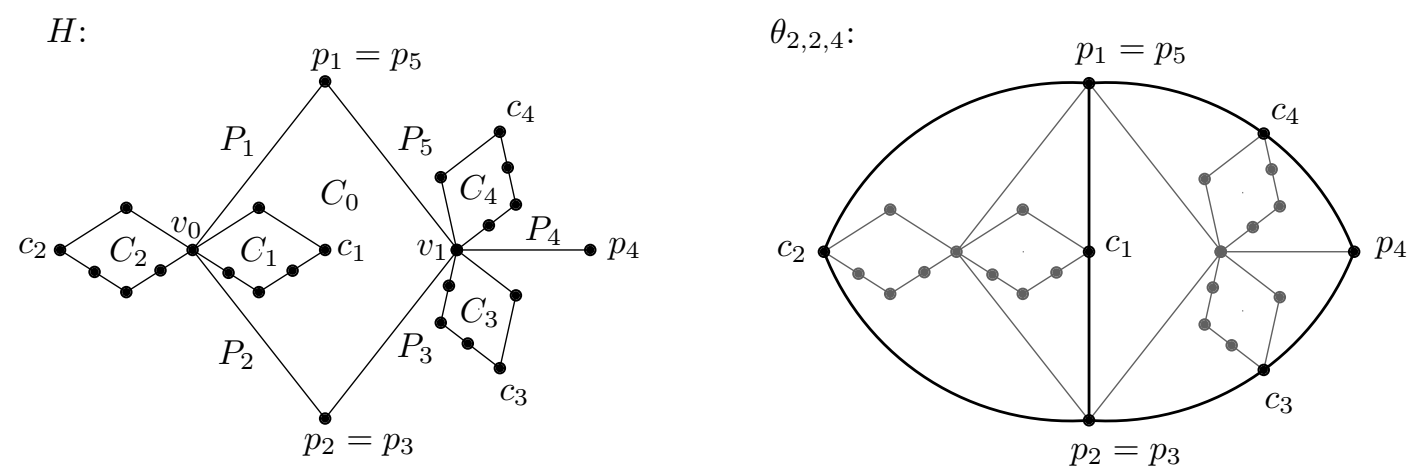

Figure 7: Graph $H$ for $j=2, k=4$ (left) and forcing $\theta_{2,2,4}$ using $H$ (right).

Observe first that having a monochromatic copy of a $H$, Builder could easily force $\theta_{2, j, k}$ (cf. Figure 7). The graph $H$ is outerplanar, and hence unavoidable by Theorem 1 . The problem is that by connecting the proper vertices of the monochromatic copy of $H$ in the resulting graph, the planarity condition would be violated. Therefore, we have to change the strategy for forcing $H$.

For $n=0, \ldots, j^{\prime}+k^{\prime}+1$, let $G_{n}:=H\left[V\left(C_{0} \cup \cdots \cup C_{n}\right)\right]$. So, the graph $G_{j^{\prime}+k^{\prime}+1}$ is the graph $H$ without the paths $P_{2}, \ldots, P_{j^{\prime}}, P_{j^{\prime}+3}, \ldots, P_{j^{\prime}+k^{\prime}+1}$. Let us refer to the blocks of $G_{n}$ and the corresponding vertices of the complete block graph simply by $C_{0}, C_{1}, \ldots, C_{n}$. Next, let $V^{\prime}$ be the set of vertices of $G_{n}$ (and thus also of $\bar{B}\left(G_{n}\right)$ ) for which the distance from $v_{0}$ in $G_{n}$ is even. For $G_{n}$, we define a subdivided complete block graph $\overline{B_{S}}\left(G_{n}\right)$ as a graph that arises from $\bar{B}\left(G_{n}\right)$ by subdividing each edge joining $C_{i}(i=1, \ldots, n)$ and a vertex of $V^{\prime}(k-1)$-times. Observe that $\overline{B_{S}}(G)$ is a tree, and that $G \cup \overline{B_{S}}(G)$ is planar.

We now present strategy $\mathbf{D}$ for forcing $G_{n}$.

\section{strategy $\mathbf{D}\left(G_{n}\right)$}

1. If $n=0$, call strategy $\mathbf{C}\left(C_{0}\right)$. In $C_{0}$, find a good path $P_{0}$, denote the middle vertex of $P_{0}$ by $v_{0}$ and its opposite vertex in $C_{0}$ by $v_{1}$.

2. If $n \geqslant 1$, let $T^{\prime}=\overline{B_{S}}\left(G_{n}\right), G^{\prime}=G_{n-1}, v^{\prime}=v_{0}$ if $n \leqslant j^{\prime}+1$ and $v^{\prime}=v_{1}$ otherwise, and $\mathbf{D}^{\prime}=\mathbf{D}\left(G_{n-1}\right)$. Call strategy $\mathbf{A}\left(T^{\prime}, G^{\prime}, v^{\prime}, \mathbf{D}^{\prime}\right)$.

3. In $\left(T^{\prime}, G^{\prime}, v^{\prime}\right)$, connect two vertices of $T^{\prime}=\overline{B_{S}}\left(G_{n}\right)$ by an edge if and only if the corresponding vertices are connected by an edge in $G_{n}$.

We show by induction on $n$ that $G_{n}$ is unavoidable by strategy $\mathbf{D}\left(G_{n}\right)$, and that every graph $D$ of $\mathcal{D}\left(G_{n}\right)$ can be embedded in the plane so that

(1) all vertices $v_{0}, v_{1}, p_{1}, p_{j^{\prime}+1}, c_{1}, \ldots, c_{n}$ belong to some face $f_{1}$, and

(2) (a) the vertices $v_{0}, p_{1}, p_{j^{\prime}+1}$ belong to some face $f_{2}$, other than $f_{1}$, or

(b) there is a path $P=p_{1} c_{1} p_{j^{\prime}+1}$ of the other color than $G_{n}$. 

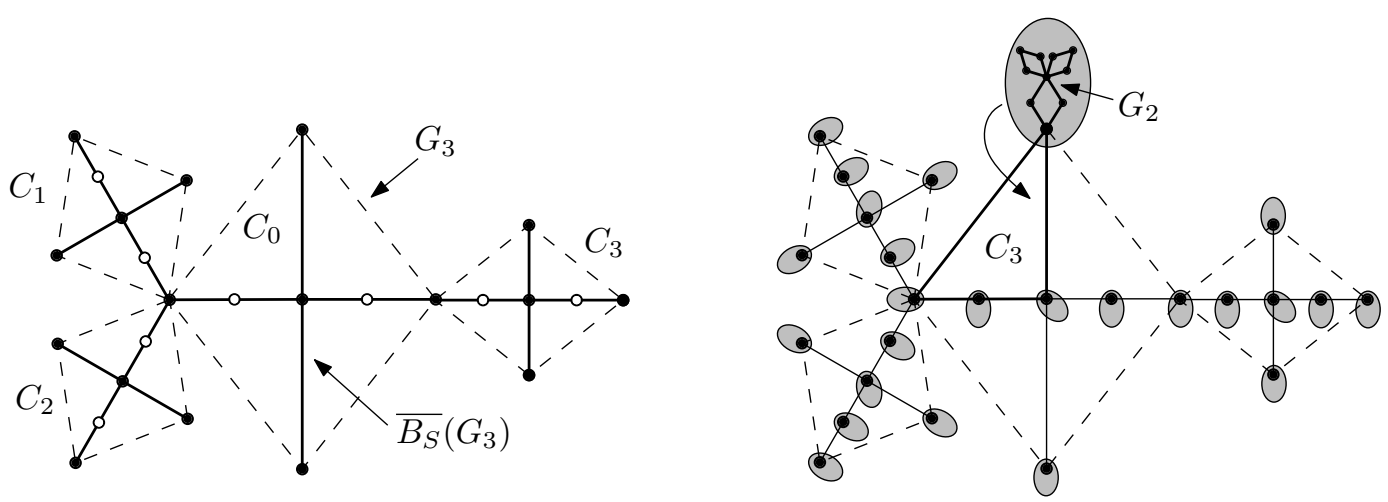

Figure 8: Left: The graphs $G_{3}$ (dashed) and $\overline{B_{S}}\left(G_{3}\right)$ (solid) for $j=2, k=2$. Black vertices represent the vertices of $\bar{B}\left(G_{3}\right)$ whereas white ones are the subdividing vertices. Right: Forcing $G_{3}$, Case 2 - one of the edges added to $\left(T^{\prime}, G^{\prime}, v^{\prime}\right)=\left(\overline{B_{S}}\left(G_{3}\right), G_{2}, v_{2}\right)$ is blue.

The base case is $n=0$. By Lemma 7 , strategy $\mathbf{C}\left(C_{0}\right)$ is a good strategy, i.e. every graph of $\mathcal{C}\left(C_{0}\right)$ can be embedded in such a way that all vertices of $C_{0}$ belong to one common face, and there is a path $P_{0} \subset C_{0}$ of length $\frac{4(j-1)}{2}=2(j-1)$ such that all vertices of $V\left(P_{0}\right)$ belong to the boundary of another face. The first part implies condition (1), and the second part implies condition $(2)(a)$.

Now assume that $n \geqslant 1$. We first show that $D$ is planar. Every graph of $\mathcal{D}\left(G^{\prime}\right)$ is planar by induction. Therefore, every graph $A$ of $\mathcal{A}\left(T^{\prime}, G^{\prime}, v^{\prime}, \mathbf{D}^{\prime}\right)$ is reducible to $T^{\prime}$ by Lemma 4, and thus planar. Since $T^{\prime}=\overline{B_{s}}\left(G_{n}\right)$, every graph that arises in Step 3 of strategy $\mathbf{D}\left(G_{n}\right)$ is reducible to $\overline{B_{s}}\left(G_{n}\right) \cup G_{n}$, which is planar.

Let us prove that a monochromatic copy of $G_{n}$ arises when following strategy $\mathbf{D}\left(G_{n}\right)$, and that $D$ fulfills Conditions (1) and (2). Suppose that the monochromatic copy of $\left(T^{\prime}, G^{\prime}, v^{\prime}\right)$ in $A$ is blue. Let us focus on the edges added in Step 3. The following two cases can arise.

Case 1: All these edges are red. Since all the edges form a copy of $G_{n}$, we get a red $G_{n}$. According to the discussion above, $D$ is reducible to $\overline{B_{s}}\left(G_{n}\right) \cup G_{n}$. Every $\overline{B_{s}}\left(C_{i}\right)$ $(i=0, \ldots, n)$ can be drawn inside the cycle $C_{i}$ of $G_{n}$. Then, all the vertices of $G_{n}$ belong to the boundary of the outer face, which gives us Condition (1). Condition (2) is also satisfied as there is a blue path $p_{1} C_{0} p_{j^{\prime}+1}$.

Case 2: At least one edge $e$ is blue. The edge $e$ joins the ends of a blue path $P$ of length $k+1$ of $T^{\prime}$. So, a cycle $C_{n}$ of length $k+2$ is formed. Since there is a copy of $G^{\prime}=G_{n-1}$ appended to each vertex of $T^{\prime}$ and $G_{n}=G_{n-1} \cup C_{n}, k+2$ blue copies of $G_{n}$ are formed. Builder arbitrarily chooses one of them, called $G_{n}$. The graph $A$ of $\mathcal{A}\left(T^{\prime}, G^{\prime}, v^{\prime}, \mathbf{D}^{\prime}\right)$ is $\left(T^{\prime}, \mathcal{D}^{\prime}\right)$-reducible by Lemma 4 . Since $P \subseteq T^{\prime}$, the graph $A$ is $\left(P, \mathcal{D}^{\prime}\right)$ reducible. Let $D^{\prime}$ be the graph of $\mathcal{D}^{\prime}$ that contains the copy of $G_{n-1}$ in the chosen blue graph $G_{n}$. By the induction hypothesis, $D^{\prime}$ can be embedded so that Conditions (1) and (2) are satisfied (for $G_{n-1}$ in $D^{\prime}$ ). Therefore, the graph $D$ can be embedded is such a way that all the vertices $v_{0}, v_{1}, p_{1}, p_{j^{\prime}+1}, c_{1}, \ldots, c_{n-1}$ of $G^{\prime}$ lie in the boundary of a common 

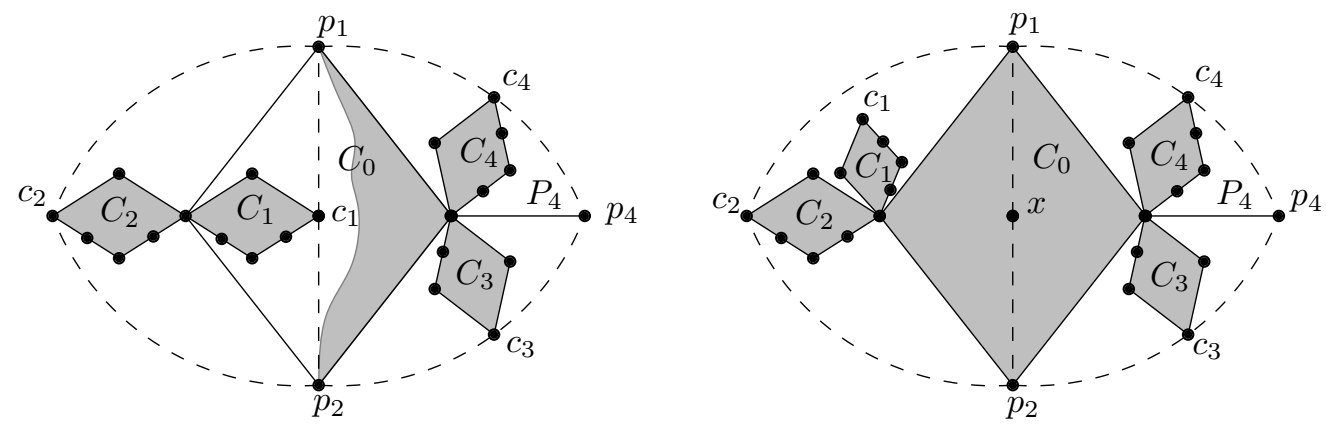

Figure 9: Adding edges of a copy of $\theta_{2,2,4}$ to $H$ if the condition (2)a (left), resp. (2)b (right) is satisfied.

face and either $v_{0}, p_{1}, p_{j^{\prime}+1}$ belong to another common face or there is red path of $p_{1} c_{1} p_{j^{\prime}+1}$. Also, $D$ can be embedded so that all vertices of $C_{n}$ lie in the boundary of a common face. Now, $G^{\prime}$ can be drawn inside that face, which gives both Condition (1) and Condition (2).

Having a monochromatic copy of $G=G_{j^{\prime}+k^{\prime}+1}$, Builder can force $H=G \oplus_{v_{0}} T_{0} \oplus_{v_{1}} T_{1}$, where $T_{0}=P_{2} \oplus_{v_{0}} \cdots \oplus_{v_{0}} P_{j^{\prime}}$ and $T_{1}=P_{j^{\prime}+3} \oplus_{v_{1}} \cdots \oplus_{v_{1}} P_{j^{\prime}+k^{\prime}+1}$. Indeed, since $G$ is unavoidable by strategy $\mathbf{D}\left(G_{j^{\prime}+k^{\prime}+1}\right)$, the supergraph $\left(T_{0}, G, v_{0}\right)$ of $G \oplus_{v_{0}} T_{0}$ is unavoidable by strategy $\mathbf{X}=\mathbf{A}\left(T_{0}, G, v_{0}, \mathbf{D}(G)\right)$ by Lemma 4 . Applying Lemma 4 again, we find that the supergraph $\left(T_{1},\left(T_{0}, G, v_{0}\right), v_{1}\right)$ of $G \oplus_{v_{0}} T_{0} \oplus_{v_{1}} T_{1}$ is unavoidable by strategy $\mathbf{A}\left(T_{1},\left(T_{0}, G, v_{0}\right), v_{1}, \mathbf{X}\right)$. In order to force $\theta_{2, j, k}$, it suffices to add the appropriate edges to $G \oplus_{v_{0}} T_{0} \oplus_{v_{1}} T_{1}$. The whole process is summed up in strategy $\mathbf{E}$.

\section{strategy $\mathbf{E}\left(\theta_{2, j, k}\right)$}

1. Call strategy $\mathbf{A}\left(T_{1},\left(T_{0}, G, v_{0}\right), v_{1}, \mathbf{X}\right)$, where $\mathbf{X}=\mathbf{A}\left(T_{0}, G, v_{0}, \mathbf{D}(G)\right)$ and $v_{1}$ is an arbitrary vertex corresponding to $v_{1}$ of some copy of $G$ in $\left(T_{0}, G, v_{0}\right)$. Chose a monochromatic copy of $H$.

2. Add edges of the cycle $p_{1} c_{2} p_{2} c_{3} \ldots p_{j^{\prime}+1}\left(=p_{j^{\prime}+2}\right) c_{j^{\prime}+2} p_{j^{\prime}+3} \ldots c_{j^{\prime}+k^{\prime}+1} p_{1}$ to $H$. If there is not the path $p_{1} c_{1} p_{j^{\prime}+1}$, also add the edges $p_{1} c_{1}$ and $c_{1} p_{j^{\prime}+1}$.

As a consequence of Lemma 4 , every graph of $\mathcal{A}\left(T_{1},\left(T_{0}, G, v_{0}\right), v_{1}, \mathbf{X}\right)$ can be embedded in such a way that Conditions (1) and (2) hold for $G$, and that all the vertices $p_{2}, \ldots, p_{j^{\prime}+k^{\prime}+1}$ lie in the boundary of the face $f_{1}$. This means that adding the cycle in Step 3 of strategy $\mathbf{E}$ does not violate the planarity of the final graph. Finally, Condition (2) ensures that either there already is a path of length 2 connecting $p_{1}$ and $p_{j^{\prime}+1}$ of the desired color, or Builder can add it by connecting $p_{1}$ to $c_{1}$ and $c_{1}$ to $p_{j^{\prime}+1}$. 


\section{$5 \quad$ Further problems}

The question of whether the class of planar graphs is self-unavoidable is still open. To disprove it, it suffices to find a single planar graph $G$ such that Painter can ensure that a monochromatic copy of $G$ never occurs when playing on planar graphs. The graph $K_{4}$ seems to be a good candidate.

Conjecture 8. $K_{4}$ is avoidable on the class of planar graphs.

Unfortunately, Painter's winning strategies seem to be much harder to find. So far, only one such strategy has been presented; namely a strategy showing that a triangle is avoidable on the class of outerplanar graphs given in [5].

\section{Acknowledgements}

The author would like to thank to Tomáš Kaiser for suggesting this problem and for his subsequent guidance. Without his help, this paper would never have been completed.

\section{References}

[1] J. Beck. On size Ramsey number of paths, trees and cycles I. J. Graph Theory, 7:115-130, 1983.

[2] J. Butterfield, T. Grauman, W. B. Kinnersley, K. G. Milans, C. Stocker, D. B. West. On-line Ramsey Theory for Bounded Degree Graphs. Electron. J. Comb., 18(11):P136, 2011.

[3] D. Conlon. On-line Ramsey numbers. SIAM J. Discrete Math., 23:1954-1963, 2009.

[4] R. Diestel. Graph Theory. Springer-Verlag, 2005.

[5] J. A. Grytczuk, M. Hałuszczak, H. A. Kierstead. On-line Ramsey Theory. Electron. J. Combin., 11(1):R57, 2004.

[6] J. A. Grytczuk, H. A. Kierstead, P. Prałat. On-line Ramsey Numbers for Paths and Stars. Discrete Math. Theor. Comp. Science, 10(3):63-74, 2008.

[7] P. E. Haxell, Y. Kohayakawa, and T. Łuczak. The induced size-Ramsey number of cycles. Combin. Probab. Comput., 4:217-239, 1995.

[8] H. A. Kierstead, G. Konjevod. Coloring number and on-line Ramsey theory for graphs and hypergraphs. Combinatorica, 29(1):49-64, 2009.

[9] A. Kurek, A. Ruciński, Two variants of the size Ramsey number, Discuss. Math. Graph Theory, 25(1-2):141-149, 2005. 